\title{
Acknowledgment to Reviewers of Future Internet in 2021
}

\section{Future Internet Editorial Office}

MDPI AG, St. Alban-Anlage 66, 4052 Basel, Switzerland

Rigorous peer-reviews are the basis of high-quality academic publishing. Thanks to the great efforts of our reviewers, Future Internet was able to maintain its standards for the high quality of its published papers. Thanks to the contribution of our reviewers, in 2021, the median time to first decision was 12 days and the median time to publication was 34 days. The editors would like to extend their gratitude and recognition to the following reviewers for their precious time and dedication, regardless of whether the papers they reviewed were finally published:

Abenza, Pedro Pablo Garrido

Abouenour, Lahsen

Abreu, Carlos

Acosta-Vargas, Patricia

Adamik, Anna

Adamski, Andrzej

Agapiou, Athos

Aguaded-Gómez, José Ignacio

Ahmed, Irfan

Aich, Satyabrata

Akser, Murat

Alani, Omar Younis

Alexandridis, Georgios

Alfeo, Antonio Luca

Alibakhshikenari, Mohammad

Citation: Future Internet Editorial

Office. Acknowledgment to

Reviewers of Future Internet in 2021.

Future Internet 2022, 14, 53. https://

doi.org/10.3390/fi14020053

Published: 10 February 2022

Publisher's Note: MDPI stays neutral with regard to jurisdictional claims in published maps and institutional affiliations.

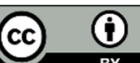

Copyright: (๑) 2022 by the author. Licensee MDPI, Basel, Switzerland. This article is an open access article distributed under the terms and conditions of the Creative Commons Attribution (CC BY) license (https://creativecommons.org/licenses/by/4.0/).
Al-Khateeb, Haider

Allani, Sabri

Almeida Mendes, Mateus Daniel

Almeida, Fernando

Almeida, Joao Emilio

Alonso, Francisco

Alonso-Muñoz, Laura

Alsharif, Mohammed H.

Amado, Miguel

Amin, Farhan

Anagnostopoulos, Marios

Anandakumar, Nalla

Andročec, Darko

Anjomshoaa, Amin

Antón, Daniel

Antunes, Mário

Antunes, Mário Joao Goncalves

Aparicio-Pardo, Ramon

Aquino, Antonio
Arai, Ayumi

Arnaiz-González, Álvar

Artiemjew, Piotr

Aslam, Sheraz

Azevedo, Fábio

Babič, František

Bach, Mirjana Pejic

Bagnoli, Franco

Bakator, Mihalj

Balducci, Fabrizio

Bankov, Dmitry

Bantimaroudis, Philemon

Barbon Junior, Sylvio

Barragán-Sánchez, Raquel

Batchkova, Idilia

Ben Haj Frej, Mohamed

Bendechache, Malika

Benítez-Andrades, Jose Alberto

Berntzen, Lasse

Bolanowski, Marek

Border Sánchez, Borja

Borza, Diana

Bossaller, Jenny

Bouchouicha, Moez

Bourgeois, Anu G.

Brad, Remus

Bresfelean, Vasile Paul

Briongos, Samira

Bures, Miroslav

Burlea-Schiopoiu, Adriana

Butusov, Denis

Buzón-García, Olga

Byeon, Haewon

Bylieva, Daria 
Cáceres Reche, Pilar

Cardoso, Jorge C. S.

Cascardi, Alessio

Castiglione, Arcangelo

Castillo-Olea, Cristian

Catak, Ferhat Ozgur

Catalin, Dumitrescu

Cauteruccio, Francesco

Cenys, Antanas

Cernian, Alexandra

Chang, Wanjung

Chaudet, Claude

Chaudhari, Meenal

Chelli, Francesco M.

Chen, Bo

Chen, Chin-Ling

Chen, Liang-Bi

Chen, Sanchuan

Cheng, Chun-An

Chergui, Hatim

Chi, Po-Wen

Chica, Jose-Maria Serrano

Chodarev, Sergej

Choudhury, Nazim

Chui, Kwok Tai

Cichosz, Paweł

Ciuc, Mihai

Coelho, Igor Machado

Colomer Feliu, Jordi

Comes, Calin-Adrian

Conde-Cespedes, Patricia

Corea, Francesco

Corti, Fabio
Dechouniotis, Dimitrios

Della Vedova, Marco L.

Delnevo, Giovanni

Demertzis, Konstantinos

Di Mauro, Mario

Díaz García, Antonio F.

Dick, Geoffrey N.

Díez-Bedmar, María Del Consuelo

Dornhöfer, Mareike

Doynikova, Elena

Du, Ke-Lin

Dumitriu, Dan

Duradoni, Mirko

Dziechciarz, Józef

Elagin, Vasiliy

Elshaw, Mark

Endo, Patricia Takako

Espelt, Ricard

Esposito, Antonio

Fallucchi, Francesca

Farmer, Lesley

Fascista, Alessio

Fattahi, Jaouhar

Fedele, Rosario

Felseghi, Raluca Andreea

Fernandes, Sandra Raquel Gonçalves

Ferreira, Ricardo

Fesenmaier, Daniel R.

Ficco, Massimo

Fidan, Ismail

Fiedler, Markus

Figueiredo, Carlos

Florea, Bogdan Cristian

Costa-Requena, Jose

Cotfas, Liviu-Adrian

Forestiero, Agostino

Gajic, Dragoljub

Cottone, Paolo

Craig, Gregory

Galdames, Patricio A.

Galić, Irena

Crisp, Geoffrey

D'Andreagiovanni, Fabio

Gao, Hongbo

García, Marcelo

D'Errico, Francesca

Da Silva Couto, Maria João Valente

García-Magariño, Iván

DaCosta, Boaventura

Damaševičius, Robertas

García-Martínez, Inmaculada

Gascón, Joan-Francesc Fondevila

Daniela, Linda

Gastaldo, Paolo

Ghosh, Joydev

Darki, Ahmad

Darwish, Mohamed

Giannakoulopoulos, Andreas

Giomelakis, Dimitrios

David, Bertrand

Gioulekas, Fotios

Davidrajuh, Reggie

De Carvalho, Rogerio Atem

Girolami, Michele

Girovský, Peter

De Giorgi, Maria Grazia 
Glowacz, Adam

Gola, Arkadiusz

Gomes, Eduardo

Gómez-Abajo, Pablo

Gomez-Jauregui, Valentin

Gómez-Rodríguez, Alma

Granda, Juan Carlos

Grobelna, Iwona

Gudelj, Anita

Guidi, Barbara

Gulmezoglu, Berk

Haghi, Mostafa

Hamza-Lup, Felix

Han, Congzheng

Han, Heesup

Hanna, Pierre

Hasan, Syed Faraz

Helebrandt, Pavol

Heo, Seonyeong

Heo, Wookjae

Heydari, Shahram

Hinas, Ajmal

Hirmer, Pascal

Ho, Danny C. K.

Hoblos, Ghaleb

Holik, Filip

Horák, Jakub

Horla, Dariusz

Hribernik, Karl

Hua, Jie

Hussain, Sajjad

Iancu, Bogdan (Finland)

Iancu, Bogdan (Romania)

Ilieva, Galina

Infante-Moro, Juan Carlos

Ingaldi, Manuela

Izquierdo-Fuente, Alberto

Izydorczyk, Jacek

Jaf, Sardar

Jakubowicz, Andrew

Jamróz, Dariusz

Jang, Gil-Jin

Jelodar, Hamed

Jeong, Dongwon

Jiménez, Jarilyn M. Hernández

Jin, Peiquan

Joshi, Gyanendra P.

Junior, Antonio

Jupowicz-Ginalska, Anna

Kabir, Russell

Kaczmarek, Jarosław
Kafle, Ved

Kakanakov, Nikolay

Kalatzis, Nikos

Kamal, Md Abdus Samad

Kamariotou, Maria

Kang, Dae-Ki

Karamitsos, Ioannis

Karampatzakis, Dimitris

Karie, Nickson M.

Karim, Ramin

Karkazis, Panagiotis

Karkazis, Panagiotis A.

Karyotis, Vasileios

Kashevnik, Alexey

Kaufmann, Michael

Kawa, Arkadiusz

Kejriwal, Mayank

Kenyeres, Martin

Keogh, John G.

Khan, Muhammad Ashfaq

Kim, Byung-Gyu

Kim, Haklae

Kim, Ki-Il

Klemens, Brygida

Koelmel, Bernhard

Kogias, Dimitris

Kolomvatsos, Kostas

Konys, Agnieszka

Korzun, Dmitry

Kosmas, Ioannis

Kotis, Konstantinos

Kotteti, Chandramouli

Kotzanikolaou, Panayiotis

Kou, Gang

Krasuski, Kamil

Kratzke, Nane

Krész, Miklós

Kröker, Ilja

Krygier, Jaroslaw

Krzystanek, Marek

Kühl, Alexander

Kuhn, Nicolas

Kuladinithi, Koojana

Kumara, Indika

Kuzminykh, Ievgeniia

Kwon, Hyun

Kyrarini, Maria

Lacroix, Agnès

Lahby, Mohamed

Lai, Cristian

Lama Ruiz, Juan Ramón 
Lazaroiu, George

Le, Thi-Thu-Huong

Lee, Cheng-Chi

Lee, Gyumyoung

Lee, Il-Gu

Lee, Ki-Sun

Lee, Manhee

Lee, Seunghwan

Lee, Tian-Fu

Levi, Itamar

$\mathrm{Li}$, Chen

Li, Chengqing

Li, Hui

$\mathrm{Li}$, Zheng (Eddie)

Lin, Han-Yu

Lin, Tsung-Hung

Lin, Yi-Bing

Listanti, Marco

Liu, Yudong

Lo Storto, Corrado

Lopes, Betina Silva

López Granado, Otoniel Mario

López, Helena Fernández

López-Borrull, Alexandre

Lopez-Martin, Manuel

Loskot, Pavel

$\mathrm{Lu}$, Ching-Ta

Luesma, María José

Lukinac, Jasmina

Lupu, Ciprian

Lytaev, Mikhail

Madokoro, Hirokazu

Madsen, Dag Øivind

Mahmood, Adnan

Makowski, Lukasz

Makris, Christos

Malinauskas, Romualdas

Maniou, Theodora

Manolova, Agata

Mantzaris, Alexander V.

Manzalini, Antonio

Manzanares-Lopez, Pilar

Marco, Hector

Marinello, Francesco

Marín-Marín, José Antonio

Marozzo, Fabrizio

Marticorena-Sánchez, Raúl

Martínez Ramón, Juan Pedro

Martínez-García, Miguel

Martínez-Otzeta, José María

Martín-Martín, Pilar
Matysik-Pejas, Renata

Mazilescu, Crisanta-Alina

Mazurek, Miroslaw

McAndrew, Ian

Meirinhos, Manuel Floindo Alves

Mellouli, Nédra

Mican, Daniel

Michailidis, Panagiotis D.

Migallón, Héctor

Mihaescu, Cristian

Mihailescu, Marius Iulian

Milicevic, Mario

Minaee, Shervin

Miralinaghi, Mohammad

Miranda, Jorge

Mitra, Rangeet

Mitraka, Elvira

Moccozet, Laurent

Moldovanu, Simona

Montisci, Augusto

Montoya, Oscar

Moon, Jihwan

Moreno Novella, Jose I.

Moreno-Marcos, Pedro Manuel

Morocho-Cayamcela, Manuel Eugenio

Moßgraber, Jürgen

Moulin, Bernard

Moustakidis, Serafeim

Muhamedyev, Ravil

Munawar, Hafiz Suliman

Muñoz, Antonio

Muñoz-Rodríguez, José Manuel

Nakatsu, Ryohei

Nazari-Heris, Morteza

Nazarkevych, Mariia

Nenkov, Nayden

Nepomuceno, Thyago

Neves, António J. R.

Nguyen, Kien

Nicolae, Mariana

Nijdam, Niels Alexander

Nikolaidis, Ioanis

Nikolakis, Nikolaos

Nkenyereye, Lewis

Nobanee, Haitham

Novikova, Evgenia

Nowostawski, Mariusz

Ntantogian, Christoforos

O'Shaughnessy, Douglas

Olivetti, Elena

Ortiz, Jordi 
Pais, Sebastião

Pallmann, Philip

Paniagua, Francisco Javier

Parra-Arévalo, María Isabel

Parrino, Stefano

Paszkiewicz, Andrzej

Patil, Madhav

Patra, Braja Gopal

Patrick, Hui Chi Leung

Patsakis, Constantinos

Pediaditis, Matthew

Peng, Zhe

Pérez Fernández, Javier

Perez-Navarro, Antoni

Perez-Ramirez, Carlos

Pescaru, Dan

Petroșanu, Dana-Mihaela

Picone, Marco

Pitropakis, Nick

Podgorelec, Blaž

Polańczyk, Andrzej

Polap, Dawid

Ponciano, Jean-Jacques

Portilla, Jorge

Poulose, Alwin

Przesmycki, Rafal

Qi, Guanqiu

Quadri, Christian

Quevedo-Blasco, Raúl

Ramanauskaitè, Simona

Ramella, Giuliana

Ramos-López, Darío

Rawski, Mariusz

Ray, Debisree

Recupero, Diego Reforgiato

Reggio, Gianna

Reijsbergen, Daniël

Reina Quintero, Antonia M.

Ren, Ju

Reyes Menéndez, Ana

Rezvani, Mohsen

Ribeiro, Jorge $\mathrm{T}$.

Righi, Rodrigo

Robitzsch, Alexander

Rodriguez-Artacho, Miguel

Rojas, Elisa

Romano, Diego

Romero, Fernando Castano

Romero-Rodríguez, José-María

Roveda, Loris

Rughinis, Razvan
Ryu, Min Ho

Saeed, Nagham

Saha, Sangeet

Saia, Roberto

Salinas, Jesus

Sánchez, Bordel

Sanchez-Gomez, Jesus

San-Segundo, Rubén

Sarasa-Cabezuelo, Antonio

Sarkar, Dipto

Sarraipa, João

Sato, Hiroyuki

Satorres, Silvia Martínez

Saura, José Ramón

Savaglio, Claudio

Scatá, Marialisa

Schoenhofer, Savina

Schöpfel, Joachim

Sebestyen, Gheorghe

Seliya, Naeem

Sengoku, Shintaro

Seredin, Oleg

Settembre-Blundo, Davide

Severino, Alessandro

Shabbir, Syed-Abdul

Shafiq, Muhammad

Sharma, Ashutosh

Sharma, Sachin

Shaukat, Kamran

Shu, Kai

Sidorov, S. P.

Silva, Edelberto Franco

Simonson, Shawn R.

Singh, Rajani

Sitar-Tăut, Dan-Andrei

Skokowski, Pawel

Slater, Luke

Smahi, Abla

Smolarz, Andrzej

Soto, Ignacio

Sousa Vieira, María Estrella

Spaanenburg, Lambert

Sperli, Giancarlo

Spiliotopoulos, Dimitris

Spoladore, Daniele

Stateczny, Andrzej

Stefanovič, Pavel

Stella, Massimo

Strzelecki, Artur

Suciu, George

Sudmanns, Martin 
Sylvia IV, J. J.

Sztangret, Izabela

Tejedor, Santiago

Teoh, Kung-Keat

Tilga, Henri

Tin, Pyke

Tintoré, Mireia

Toldinas, Jevgenijus

Tomaiuolo, Michele

Tomaszek, Katarzyna

Torres Vega, Maria

Torres, Sergio

Trakadas, Panagiotis

Tran, Gia Khanh

Troussas, Christos

Tsai, Ming-Fong

Tsigopoulos, Andreas D.

Tsunenori, Mine

Uchida, Osamu

Urrutia, Manuel Leon

Vakhania, Nodari

Van Hove, Leo

Vand, Behrang

Vardasca, Ricardo

Varga, Pal

Vasilateanu, Andrei

Vergidis, Kostas
Verma, Chaman

Véstias, Mário

Visconti, Paolo

Vlachopoulos, Dimitrios

Wang, Cheng

Wang, Shidan

Weinberger, Markus

Wnęk-Gozdek, Joanna

Wojnakowski, Marcin

Wołk, Krzysztof

Wróbel, Krzysztof

$\mathrm{Wu}$, Liwei

Wu, Ning

Wytrębowicz, Jacek

Xiao, Liangliang

$\mathrm{Xu}$, Hailu

Yang, Baijian

Yoo, Changsok

Yousif, Jabar H.

Zago, Mattia

Zalipynis, Ramon Antonio Rodriges

Zarakovitis, Charilaos

Zennaro, Marco

Zhang, Hongbo

Zhou, Mingliang

Zia, Kashif

Zlatolas, Lili Nemec 